\title{
Investigation of Blanking Nonlinearity in OFDM Systems
}

\author{
Ulrich Epple \\ Kamel Shibli \\ Michael Schnell \\ Institute of Communications and Navigation Karlsruhe Institute for Technology Institute of Communications and Navigation \\ German Aerospace Center (DLR) \\ Email: kamel.shibli@student.kit.de \\ German Aerospace Center (DLR) \\ Email: ulrich.epple@dlr.de \\ Email: michael.schnell@dlr.de
}

\begin{abstract}
In this paper, we analyze the influence of a blanking nonlinearity onto OFDM systems in detail. We will derive closedform expressions for the signal degradation and the interference, which is introduced by the blanking nonlinearity. The theoretical results will be validated by simulations, which illustrate the detrimental blanking influence in a descriptive way. In addition, it will be shown how the results affect an OFDM receiver and how the receiver can be adapted to the blanking nonlinearity. Simulation results confirm a gain of $3 \mathrm{~dB}$ at $\mathbf{B E R}=1 \cdot 10^{-3}$ for a 16QAM transmission, when adapting the receiver accordingly.
\end{abstract}

\section{INTRODUCTION}

In the past years, orthogonal frequency-division multiplexing (OFDM) has been established as a powerful and spectrally efficient modulation technique that is employed in numerous systems for wireless and wireline communications like digital audio broadcasting (DAB) [1], digital video broadcasting (DVB) [2], and powerline communications (PLC) [3]. All of these systems are often exposed to impulsive interference, e.g. originating from switching processes on the power distribution network or from the ignitions of passing vehicles, leading to a degradation of the system performance [4]. The interference situation is even more severe for the recently proposed candidate for the future L-band Digital Aeronautical Communications System (L-DACS1) [5].

For a moderate impulsive interference power and infrequent occurrence, OFDM systems can cope well with the interference, as it is spread among several subcarriers of an OFDM symbol. However for frequent appearance or high interference power, this spread will turn into a disadvantage [6] and interference mitigation techniques have to be implemented.

Conventional approaches to mitigate the impact of pulsed interference are based on applying a memoryless nonlinearity at the receiver input, prior to the conventional OFDM demodulator. The most common approaches are blanking or clipping nonlinearities as well as combined blanking-clipping nonlinearities [7]-[9].

In our investigations we will focus on the blanking nonlinearity $(\mathrm{BN})$. Since we will investigate the effects of the $\mathrm{BN}$ but not the capabilities of the $\mathrm{BN}$ itself, we will apply no impulsive noise channel statistics [10], as it has been done e.g. in [8]. This means we assume that in each OFDM symbol a known number of samples are affected by impulsive noise and all affected samples are detected and blanked. This will help us to obtain reliable results about the performance of the BN, depending on the number of blanked samples. Given this premise, the influence of the BN onto the OFDM signal is investigated in detail and described mathematically. The results will be compared to the results obtained by the extended Bussgang theorem [11], which is a general expression for nonlinear influence. It will be shown that the $\mathrm{BN}$ can be described by an equivalent distortion term, which makes it easy to predict the capabilities of the OFDM system for an expected fraction of blanks in the received signal.

Next to this theoretical analysis, we will verify our assumptions and derivations by simulations in Section IV, which will show the good match of the equivalent noise term. In Section V, we will finally demonstrate how the obtained results can be employed to adapt OFDM receiver parts such as the channel estimation block or the demodulation block, which depend on channel statistics. Simulations will emphasize that remarkable gains are achieved by adapting the receiver.

\section{SySTEM MODEL}

For our investigation, we focus on a digital baseband model. Consider the system model from Fig. 1. The information bits $I_{k}$ are coded by a rate $r=1 / 2$ convolutional coder to obtain $U_{k}$ and then mapped onto the symbols $S_{k}$. For modulation, quadrature phase shift keying (QPSK) and quadrature amplitude modulation (QAM) are used. The subsequent OFDM modulator transforms $N$ of these modulated symbols, i.e. the column vector $\mathbf{S}=\left(S_{0}, S_{1}, \ldots, S_{N-1}\right)^{T}$ into the time domain via an $N$-point inverse Discrete Fourier Transform (iDFT) and one obtains $\mathbf{s}=\left(s_{0}, s_{1}, \ldots, s_{N-1}\right)^{T}$, which can be written mathematically

$$
\mathbf{s}=\mathcal{F}^{-1} \cdot \mathbf{S}
$$

with the inverse Fourier matrix defined by

$$
\mathcal{F}_{l, k}^{-1}=\frac{1}{\sqrt{N}} e^{2 \pi j \frac{k l}{N}}, \quad l, k=0, \ldots, N-1,
$$

with $k$ being the subcarrier index in the frequency domain and $l$ denoting the sample index in the time domain. A received signal sample $r_{l}$ is obtained by adding an additive white Gaussian noise sample $n_{l}$

$$
r_{l}=s_{l}+n_{l}, \quad l=0, \ldots, N-1 .
$$




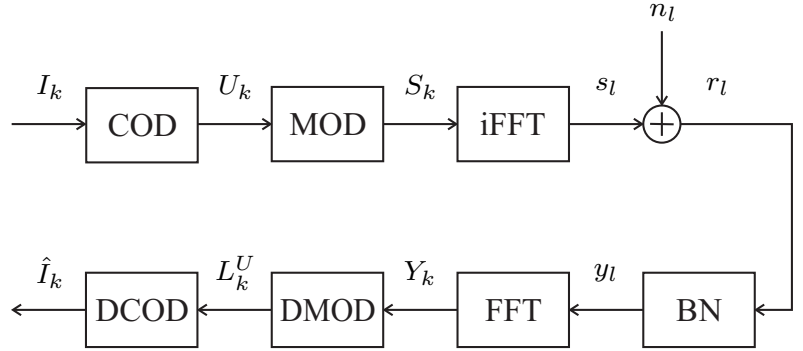

Fig. 1. System Model for OFDM transmission with blanking nonlinearity.

The power of the transmitted signal is normalized to 1 , i.e. $E\left\{\left|s_{l}\right|^{2}\right\}=2 \sigma_{s}^{2}=1$ and for the power of the AWGN holds $N_{0}=2 \sigma_{n}^{2}$, with $\sigma_{s}^{2}$ and $\sigma_{n}^{2}$ being the component-wise variance of the respective signal.

At the receiver, a digital $\mathrm{BN}$ is applied to the signal in the time domain. In our investigation, we blank a fixed number $N_{\mathcal{B}}$ of samples in each OFDM symbol, which complies with the case that $N_{\mathcal{B}}$ impulsive noise samples occur in an OFDM symbol, which are perfectly detected and blanked. This is, strictly speaking, not a nonlinearity, as the blanks are chosen randomly and not depending on the amplitude, it is rather the emulation of a blanking nonlinearity. This will help us to quantify the effects of the BN. The positions of the blanks within an OFDM symbol are chosen randomly. The signal after the BN is denoted by $y_{l}$. The blanking block is followed by an OFDM demodulator for transforming the signal into the frequency domain via an $N$-point DFT and obtaining $Y_{k}$. The demodulator at the next stage calculates log-likelihood ratios (LLR) $L_{k}^{U}$ for the coded bits which are fed into the decoder for obtaining estimates $\hat{I}_{k}$ of the transmitted information bits.

\section{INFLUENCE OF BLANKING NONLINEARITY}

The impact of the BN on OFDM systems had been investigated in [12]. Accordingly, the blanking will be described by a windowing operation. The window function $b_{l}$ is a sampled rectangular window that exhibits notches at those positions $l \in \mathcal{B}$ where the received signal is blanked. The number of blanks in an OFDM symbol is given by $N_{\mathcal{B}}$. Mathematically this is described by

$$
y_{l}=r_{l} \cdot b_{l}
$$

with

$$
b_{l}= \begin{cases}0 & l \in \mathcal{B} \\ 1 & \text { else. }\end{cases}
$$

This multiplication with a rectangular window leads to a convolution with the corresponding spectrum in the frequency domain. If no blanking occurs in an OFDM symbol, the window length is exactly the DFT length $N$, leading to a convolution with a sampled si-function with a one at the sampling index zero and zeros at the other samples, when the sampling grid is normalized to the subcarrier spacing. When blanking $N_{\mathcal{B}}$ arbitrary samples, the rectangular window is subdivided into multiple windows, each comprising less samples than the original one. This leads to wider si-functions, with non-zero values at all samples. Thus the orthogonality between the subcarriers gets lost and inter-carrier interference (ICI) is induced. In addition, the amplitude at the sampling index zero is attenuated, i.e. the useful signal power is reduced. This concrete description is mathematically described by the convolution of the received signal in the frequency domain $R_{k}$ with the Fourier transform of the blanking window $B_{k}$

$$
\begin{aligned}
Y_{k} & =\frac{1}{\sqrt{N}}\left(R_{k} * B_{k}\right) \\
& =\frac{1}{\sqrt{N}} \sum_{p=0}^{N-1} R_{p} B_{(k-p) \bmod N} \\
& =\frac{1}{\sqrt{N}} \sum_{p=0}^{N-1}\left(S_{p}+N_{p}\right) B_{(k-p) \bmod N}
\end{aligned}
$$

using the Fourier transform of (3). Next, we will split this equation into a part containing useful information about the $k$ th subcarrier, called $S_{k}^{\prime}$, and a part containing noise and the induced ICI, which will be called distortion term $D_{k}$ in the following,

$$
Y_{k}=S_{k}^{\prime}+D_{k}
$$

As the modulated symbols at different subcarriers are statistically independent, $S_{k}^{\prime}$ only depends on $S_{k}$ and we can write

$$
S_{k}^{\prime}=\frac{1}{\sqrt{N}} S_{k} B_{0}
$$

According to (2), we define the Fourier transform by a multiplication with a matrix, which entries are defined by

$$
\mathcal{F}_{k, l}=\frac{1}{\sqrt{N}} e^{-2 \pi j \frac{k l}{N}}, \quad k, l=0, \ldots, N-1 .
$$

Applied to the blanking window $\mathbf{b}=\left(b_{0}, b_{1}, \ldots, b_{N-1}\right)^{T}$ we obtain

$$
\mathbf{B}=\mathcal{F} \cdot \mathbf{b},
$$

with $\mathbf{B}=\left(B_{0}, B_{1}, \ldots, B_{N-1}\right)^{T}$. Given this equation and keeping (5) in mind, we obtain immediately

$$
B_{0}=\frac{N-N_{\mathcal{B}}}{\sqrt{N}}
$$

and finally for $S_{k}^{\prime}$

$$
S_{k}^{\prime}=\frac{1}{\sqrt{N}} S_{k}\left(\frac{1}{\sqrt{N}}\left(N-N_{\mathcal{B}}\right)\right)=K \cdot S_{k},
$$

with

$$
K=\left(1-\frac{N_{\mathcal{B}}}{N}\right)
$$

That means, the blanking leads to an attenuation of the useful signal $S_{k}$, without changing its phase, since $K$ is a real factor.

For $D_{k}$, we obtain

$$
D_{k}=K \cdot N_{k}+\frac{1}{\sqrt{N}} \sum_{\substack{p=0 \\ p \neq k}}^{N-1}\left(S_{p}+N_{p}\right) B_{(k-p) \bmod N} .
$$


Given a sufficient large number of subcarriers, we can assume a Gaussian distribution for $D_{k}$. For determining the variance of $D_{k}$, we first have to determine $E\left\{|\mathbf{B}|^{2}\right\}$. With (10) it follows

$$
E\left\{|\mathbf{B}|^{2}\right\}=E\left\{|\mathcal{F} \cdot \mathbf{b}|^{2}\right\}=\mathcal{F} E\left\{\mathbf{b b}^{H}\right\} \mathcal{F}^{-1} .
$$

Keeping in mind that the blanks are chosen randomly, it becomes obvious that all entries on the main diagonal of the matrix $E\left\{\mathbf{b b}^{H}\right\}$ are identical, as well as all other entries are different, but constant. This writes mathematically

$$
E\left\{\mathbf{b b}^{H}\right\}=c \cdot \mathbf{1}+d \cdot \mathbf{I}
$$

with the matrix of ones $\mathbf{1}$ and the identity matrix I. The maindiagonal values $c+d$ correspond to the probability that a sample is not blanked, as in the blanking case, $b_{l}$ is zero and does not contribute to the expectation value. Thus we get

$$
c+d=\frac{N-N_{\mathcal{B}}}{N} \text {. }
$$

With the same argumentation, the non-diagonal entries correspond to the probability that two arbitrary samples are not blanked, which writes mathematically

$$
c=\frac{\left(\begin{array}{l}
2 \\
2
\end{array}\right) \cdot\left(\begin{array}{c}
N-2 \\
N-N_{\mathcal{B}}-2
\end{array}\right)}{\left(\begin{array}{c}
N \\
N-N_{\mathcal{B}}
\end{array}\right)}=\frac{\left(N-N_{\mathcal{B}}\right)\left(N-N_{\mathcal{B}}-1\right)}{N(N-1)}
$$

and consequently

$$
d=\frac{\left(N-N_{\mathcal{B}}\right) N_{\mathcal{B}}}{N(N-1)} .
$$

This helps us to calculate $E\left\{|\mathbf{B}|^{2}\right\}$

$$
E\left\{|\mathbf{B}|^{2}\right\}=c\left(\begin{array}{cccc}
N & 0 & \ldots & 0 \\
0 & 0 & \ldots & 0 \\
\vdots & \vdots & \ddots & \vdots \\
0 & 0 & \ddots & 0
\end{array}\right)+d \cdot \mathbf{I}
$$

leading to

$$
E\left\{\left|B_{k}\right|^{2}\right\}=d=\frac{\left(N-N_{\mathcal{B}}\right) N_{\mathcal{B}}}{N(N-1)}, \quad k \neq 0 .
$$

Now we are able to calculate the variance $\operatorname{Var}\left(D_{k}\right)$, when keeping in mind that the different noise contributions $N_{k}$ and modulated symbols $S_{k}$ are uncorrelated. Besides, the signal power is normalized to one, i.e. $E\left\{\left|S_{k}\right|^{2}\right\}=1$ and the noise power is $E\left\{\left|N_{k}\right|^{2}\right\}=2 \sigma_{n}^{2}$ and we obtain

$$
\begin{aligned}
\operatorname{Var}\left(D_{k}\right) & =K^{2} 2 \sigma_{n}^{2}+\frac{N-1}{N}\left(1+2 \sigma_{n}^{2}\right) \frac{\left(N-N_{\mathcal{B}}\right) N_{\mathcal{B}}}{N(N-1)} \\
& =K^{2} 2 \sigma_{n}^{2}+(1-K) K\left(1+2 \sigma_{n}^{2}\right) .
\end{aligned}
$$

Finally this allows us to derive a closed expression for the SNR of $Y_{k}$ in (7) after the BN

$$
\mathrm{SNR}_{\mathrm{BN}}=\frac{\operatorname{Var}\left(S_{k}^{\prime}\right)}{\operatorname{Var}\left(D_{k}\right)}=\frac{K^{2}}{K(1-K)+2 \sigma_{n}^{2} K} .
$$

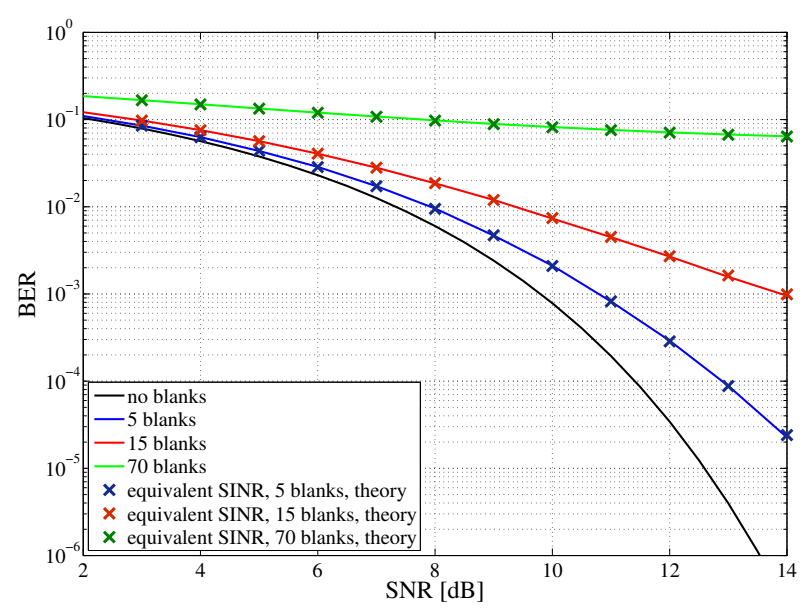

Fig. 2. BER curves for different number of blanks for an uncoded transmission.

The derived attenuation factor $K$ is not equal to the scaling factor $K_{0}$, which can be obtained by applying the extended Bussgang theorem [11]. The definition of $K_{0}$ is

$$
K_{0}=\frac{E\left\{y_{l} s_{l}^{*}\right\}}{E\left\{\left|s_{l}\right|^{2}\right\}}
$$

That means that $K_{0}$ is a constant expectation value, relying on sufficient knowledge about the received signal, i.e. about the impulsive noise channel. For the BN, it was derived in [8]. In contrary, $K$ may vary from OFDM symbol to OFDM symbol and can be calculated deterministically, only by looking at the number of blanked samples. The consequence is that $K_{0}$ can be used to estimate the average performance loss of an OFDM system, exposed to impulsive noise with known statistics, whereas $K$ is a factor which can be employed for adapting the OFDM receiver to the number of blanks, for each OFDM symbol separately, as it will be explained later on.

\section{Simulation Results}

In this section, we will verify our assumptions concerning the SNR after the BN by means of simulations. Especially, the assumed Gaussian distribution of the distortion term $D_{k}$ has to be confirmed by simulations, as this assumption is only valid for a sufficient large number of subcarriers and randomly chosen blanks. In Fig. 2, bit-error-rate (BER) curves for an uncoded transmission via an AWGN channel are plotted. For the transmission, $N=256$ and QPSK modulation was chosen. Beside the curves for a different number of blanks per OFDM symbol, theoretical results are depicted. The theoretical values were derived by a transmission over an AWGN channel with the equivalent $\mathrm{SNR}_{\mathrm{BN}}$ given in (23) without any blanking. The results confirm our assumptions concerning the distribution and the variance of $D_{k}$, as the simulated curves match the theoretical values very well for different number of blanks per OFDM symbol. It is also remarkable that even for a very small fraction of blanks, in our case $5 / 256$, the degradation is already $1 \mathrm{~dB}$ at $\mathrm{BER}=1 \cdot 10^{-3}$. 


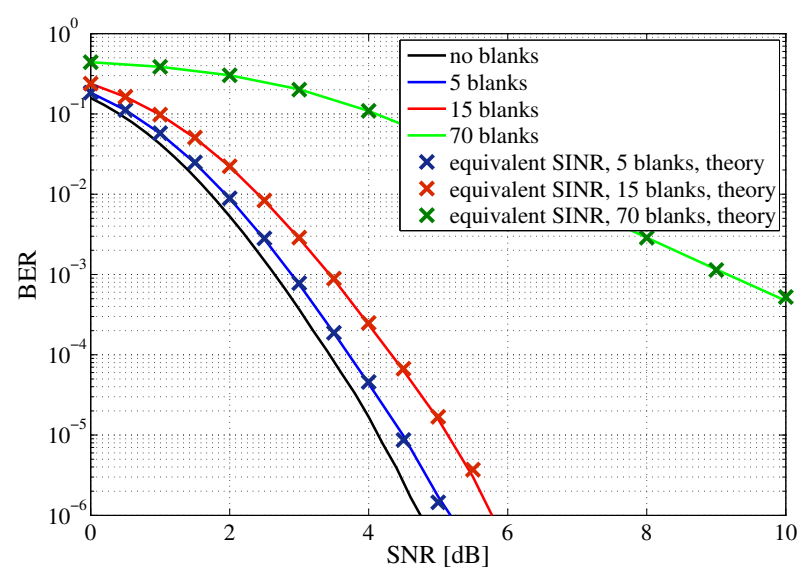

Fig. 3. BER curves for different number of blanks for a coded transmission.

The same simulations have been performed for a coded transmission with a $r=1 / 2$ convolutional code. The curves in Fig. 3 show a similar behavior in comparison to the uncoded ones, the theoretical curves fit well again. However it points out, that for a small number of blanks, the SNR loss is much smaller than in the uncoded case. Considering again $\mathrm{BER}=1 \cdot 10^{-3}$ with blanking a fraction of $5 / 256$, the degradation is only $0.3 \mathrm{~dB}$. This becomes clear by having a closer look at (23). For a coded transmission, acceptable BER are achieved for much smaller SNR values, i.e. higher noise powers compared to the uncoded transmission. In this case the denominator in (23) is governed by the noise term, as it is large compared to $K(1-K)$. For higher SNR regions, like for the uncoded transmission when looking at the same BER, the ICI part $K(1-K)$ becomes significant and leads to greater losses in the SNR.

Fig. 4 highlights this effect, where the SNR loss is plotted versus the SNR and versus the number of blanks for $N=256$. For low SNR values, the SNR loss increases nearly linear for a rising number of blanks, the slope is given by $K$ when neglecting the ICI part $K(1-K)$ in the denominator of (23). For high SNR values, this factor cannot be neglected any longer, leading to a non-linear loss of the SNR with high losses even when blanking only few samples. This result stresses the importance for achieving low SNR working points when designing systems with $\mathrm{BN}$ to cope with expected impulsive interference, e.g. by applying a strong channel coding.

\section{AdAPTION OF RECEIVER}

Beside the easy prediction of the performance with $\mathrm{BN}$, the closed-form expression for the modified $\mathrm{SNR}_{\mathrm{BN}}$ helps us to adapt our receiver to the BN. First, the demodulation is affected. As the useful signal $S_{k}$ is attenuated by the factor $K$, the received symbols are shifted with respect to the decision boundaries of the modulation scheme. For QPSK this makes no difference, as the information is contained only in the phase, and the multiplication with the real factor $K$ induces no phase shift. However, when thinking about higher order modulation, e.g. 16QAM, the received symbols after the BN

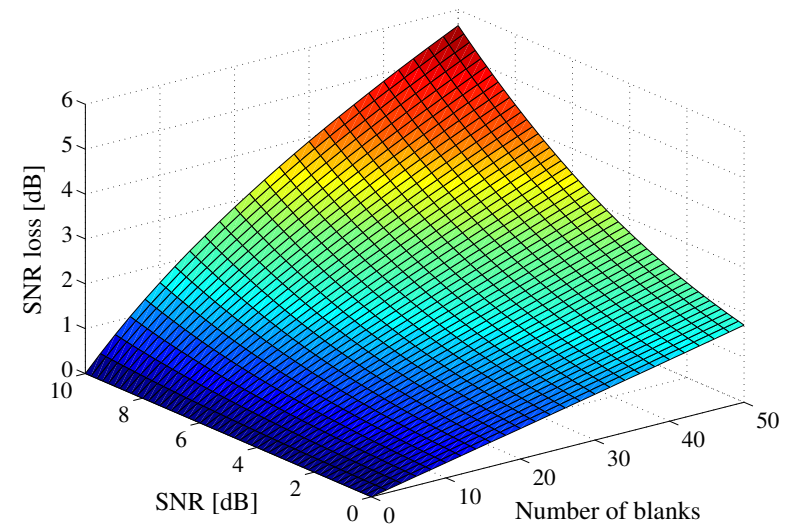

Fig. 4. SNR loss depending on the SNR and number of blanks for $N=256$.

have to be scaled to the constellation diagram. When applying a soft demodulation, mostly in terms of calculating LLRs for the coded bits, the modified noise variance has to be taken into account as well, as the calculation of the LLRs depends on the probability density function for the transmitted symbols $S_{k}$, given the received symbols $R_{k}$

$$
p\left(S_{k} \mid R_{k}\right)=\frac{1}{2 \pi \sigma_{n}^{2}} \cdot \exp \left(\frac{-\left|R_{k}-S_{k}\right|^{2}}{2 \sigma_{n}^{2}}\right) .
$$

When adapting the receiver, the noise power $2 \sigma_{n}^{2}$ has to be replaced by the distortion power $\operatorname{Var}\left(D_{k}\right)$, which follows also a Gaussian distribution. In addition, the received symbols $R_{k}$ have to be replaced by the symbols $Y_{k}$ after the BN and the constellation points $S_{k}$ alter to the attenuated ones $S_{k}^{\prime}$. This leads to the adjusted probability density function

$p\left(S_{k}^{\prime} \mid Y_{k}\right)=\frac{1}{K(1-K)+2 \pi \sigma_{n}^{2} K} \cdot \exp \left(\frac{-\left|Y_{k}-K \cdot S_{k}\right|^{2}}{K(1-K)+2 \sigma_{n}^{2} K}\right)$.

Especially for heavily varying numbers of blanks from OFDM symbol to OFDM symbol, this incorporation of the modified noise will lead to correct reliabilities for the obtained LLRs, depending on the number of blanks per OFDM symbol.

The beneficial influence of adapting the demodulation is depicted in Fig. 5. To incorporate the effect of shifting the constellation diagram, 16QAM was applied as modulation scheme. For covering the effect of correct reliabilities for the different LLRs, the number of blanks in an OFDM symbol varies between $N_{\mathcal{B}}=0, \ldots, 50$ blanks, distributed uniformly. Again, $N=256$, a transmission over an AWGN channel and channel coding with $r=1 / 2$ has been applied. Especially at low BER the beneficial influence of adapting the demodulation turns out. While at BER $=1 \cdot 10^{-2}$, the gain when adapting the demodulation is only $1 \mathrm{~dB}$, at $\mathrm{BER}=1 \cdot 10^{-3}$ a gain of $3 \mathrm{~dB}$ can be achieved by adapting the receiver.

For realistic transmission channels, a channel estimation scheme like Wiener filtering is common. In our considerations, it is split up into an interpolation in time direction and a subsequent interpolation in frequency direction. Wiener filtering 


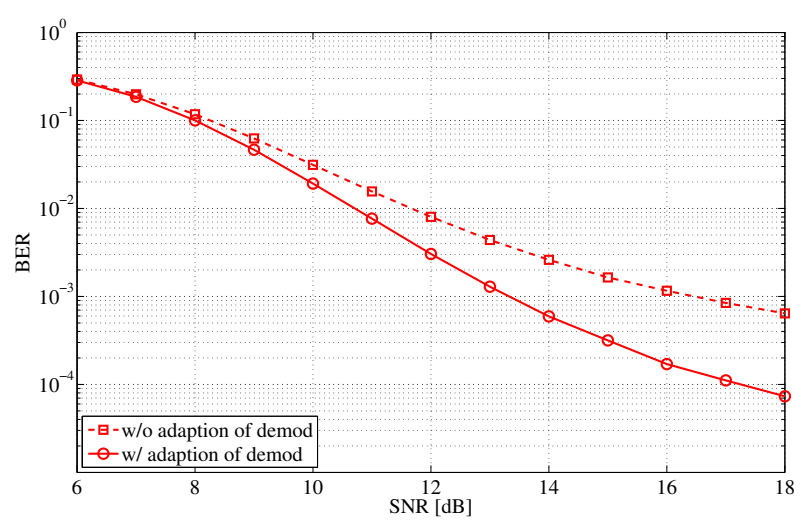

Fig. 5. Influence of adapting the demodulation to the blanking nonlinearity.

relies on the knowledge of the channel statistics [13] [14]. Given the channel statistics, one can derive the auto-covariance function $\mathbf{R}_{\tilde{H} \tilde{H}}$ between the different pilot symbol positions $l^{\prime}$. For the interpolation in time direction, it is given by

$$
\mathbf{R}_{\tilde{H} \tilde{H}}=\left(\begin{array}{ccc}
R_{t ;\left(l_{1}^{\prime}-l_{1}^{\prime}\right)}+1 / \mathrm{SNR} & \ldots & R_{t ;\left(l_{1}^{\prime}-l_{P_{t}}^{\prime}\right)} \\
\vdots & \ddots & \vdots \\
R_{t ;\left(l_{P_{t}}^{\prime}-l_{1}^{\prime}\right)} & \ldots & R_{t ;\left(l_{P_{t}}^{\prime}-l_{P_{t}}^{\prime}\right)}+1 / \mathrm{SNR}
\end{array}\right) .
$$

Again, the regular SNR can be replaced by the modified $\mathrm{SNR}_{\mathrm{BN}}$ after the $\mathrm{BN}$ to improve the results of the channel estimation as well. However, one should keep in mind that the distortion $D_{k}$ for different subcarriers in one OFDM symbol is no longer uncorrelated, as the $\mathrm{BN}$ affects all subcarriers in the same way, which has to be taken into account. For the interpolation in frequency direction, this will lead to dependencies of the distortion at different pilot symbols, i.e. contributions of $\mathrm{SNR}_{\mathrm{BN}}$ not only on the main diagonal of (27). It does not affect the interpolation in time direction, as the blanking in one OFDM symbol does not depend on the other OFDM symbols. In Fig. 6 the MSE of the channel estimation is given for Wiener filtering and a typical twopath scenario, comprising a strong line-of-sight path and an attenuated, reflected path. It becomes obvious that adapting the SNR in the CE significantly improves the performance, especially for high SNR values.

\section{CONCLUSION}

In this paper we investigated the influence of a blanking nonlinearity onto OFDM systems. First, we derived closedform expressions for the influence onto the useful OFDM signal as well as for the induced interference by the blanking nonlinearity. The final outcome of the investigation was a term for the SNR of an equivalent transmission without blanking. These results were compared with the extended Bussgang's theorem, exposing the differences between both approaches. In a second step, we confirmed the validity of the equivalent SNR by means of simulation, which show a good match with our theoretical results. Subsequently, we had a look at the impact of the blanking nonlinearity on the receiver and we showed

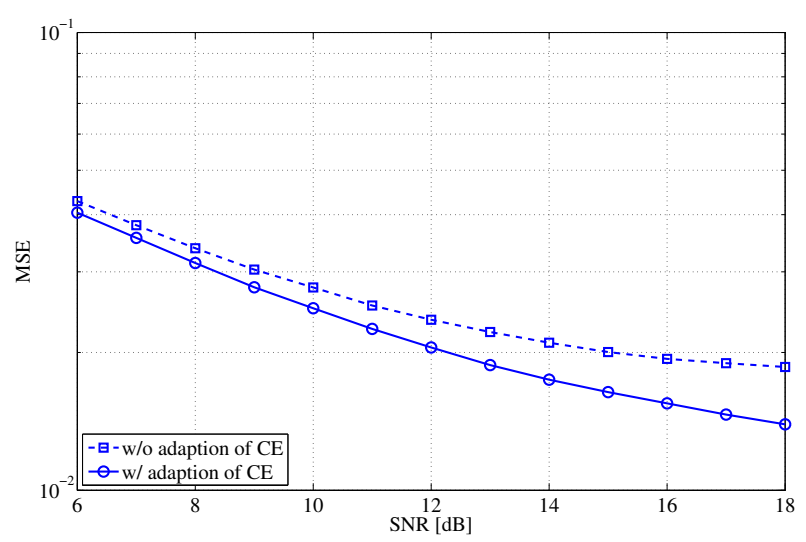

Fig. 6. Influence of adapting the channel estimation to the blanking nonlinearity.

how the receiver has to be adapted. Finally, the beneficial influence of this adaption was validated by means of BER and MSE simulations.

\section{REFERENCES}

[1] European Telecommunications Standards Institute (ETSI), "Radio Broadcasting Systems; Digital Audio Broadcasting (DAB) to mobile, portable and fixed receivers," 2000, ETSI EN 300401.

[2] European Telecommunications Standards Institute (ETSI), "Digital Video Broadcasting (DVB); Framing structure, channel coding and modulation for digital terrestrial television," September 2008, EN 300 744, Version 1.6.1.

[3] IEEE, "1901 Draft Standard for Broadband over Power Line Networks: Medium Access Control and Physical Layer Specifications," January 2010.

[4] Y.H. Ma, P.L. So, and E. Gunawan, "Performance analysis of OFDM systems for broadband power line communications under impulsive noise and multipath effects," IEEE Transactions on Power Delivery, vol. 20, no. 2, pp. 674-682, April 2005

[5] M. Sajatovic, B. Haindl, M. Ehammer, Th. Gräupl, M. Schnell, U. Epple, and S. Brandes, "L-DACS1 System Definition Proposal: Deliverable D2," Tech. Rep., Eurocontrol, Issue 1.0, February 2009.

[6] M. Gosh, "Analysis of the effect of impulse noise on multicarrier and single carrier QAM systems," IEEE Transactions on Communications, vol. 4, no. 2, pp. 145-147, February 1996.

[7] Grace Xingxin Gao, "DME/TACAN Interference and its Mitigation in L5/E5 Bands," in ION Institute of Navigation Global Navigation Satellite Systems Conference, Fort Worth, TX, USA, September 2007.

[8] Sergey V. Zhidkov, "Analysis and Comparison of Several Simple Impulsive Noise Mitigation Schemes for OFDM Receivers," IEEE Transactions on Communications, vol. 56, no. 1, pp. 5-9, January 2008.

[9] H. A. Suraweera, C. Chai, J. Shentu, and J. Armstrong, "Analysis of impulse noise mitigation techniques for digital television systems," in Proc. 8th International OFDM Workshop, September 2003, pp. 172176.

[10] S. A. Kassam, Signal Detection in Non-Gaussian Noise, SpringerVerlag, Berlin, Germany, 1988.

[11] V. Tralli D. Dardari and A. Vaccari, "A theoretical characterization of nonlinear distortion effects in OFDM systems," IEEE Transactions on Communications, vol. 48, no. 10, pp. 1755-1763, October 2000.

[12] S. Brandes, U. Epple, and M. Schnell, "Compensation of the Impact of Interference Mitigation by Pulse Blanking in OFDM Systems," in IEEE Globecom 2009 Signal Processing for Communications Symposium, Honululu, HI, USA, November 2009.

[13] P. Hoeher, S. Kaiser, and P. Robertson, "Two-dimensional pilot-symbolaided channel estimation by wiener filtering," in Proc. IEEE ICA SSP, Munich, Germany, April 1997, pp. 1845-1848.

[14] S. Jelting F. Sanzi and J. Speidel, "A comparative study of iterative channel estimators for mobile OFDM systems," IEEE Transactions on Wireless Communications, pp. 849-859, September 2003. 